\title{
Effect of Jet Inclination Angle and Hole Exit Shape on Vortical Flow Structures in Low-Reynolds Number Jet in Cross-Flow
}

\author{
Yufeng Yao, ${ }^{1}$ Mohamad Maidi, ${ }^{1}$ and Jun Yao $^{2}$ \\ ${ }^{1}$ School of Aerospace and Aircraft Engineering, Kingston University, London SW15 3DW, UK \\ ${ }^{2}$ School of Engineering, University of Lincoln, Brayford Pool, Lincoln LN6 7TS, UK \\ Correspondence should be addressed to Yufeng Yao, y.yao@kingston.ac.uk
}

Received 30 April 2012; Revised 20 July 2012; Accepted 23 July 2012

Academic Editor: S. Taib

Copyright ( 2012 Yufeng Yao et al. This is an open access article distributed under the Creative Commons Attribution License, which permits unrestricted use, distribution, and reproduction in any medium, provided the original work is properly cited.

\begin{abstract}
Numerical studies have been performed to visualize vortical flow structures emerged from jet cross-flow interactions. A single square jet issuing perpendicularly into a cross-flow was simulated first, followed by two additional scenarios, that is, inclined square jet at angles of $30^{\circ}$ and $60^{\circ}$ and round and elliptic jets at an angle of $90^{\circ}$, respectively. The simulation considers a jet to cross-flow velocity ratio of 2.5 and a Reynolds number of 225, based on the free-stream flow quantities and the jet exit width in case of square jet or minor axis length in case of elliptic jet. For the single square jet, the vortical flow structures simulated are in good qualitative agreement with the findings by other researchers. Further analysis reveals that the jet penetrates deeper into the cross-flow field for the normal jet, and the decrease of the jet inclination angle weakens the cross-flow entrainment in the near-wake region. For both noncircular and circular jet hole shapes, the flow field in the vicinity of the jet exit has been dominated by large-scale dynamic flow structures and it was found that the elliptic jet hole geometry has maximum "lifted-off" effect among three hole configurations studied. This finding is also in good qualitative agreement with existing experimental observations.
\end{abstract}

\section{Introduction}

The problem of injecting fluid through pipe/duct geometry into a mainstream cross-flow domain presents in many industrial and engineering applications, for example, turbine blade film cooling, fuel injection in IC engine, thrust, and noise control of S/VTOL aircraft, fuel-air mixing in gas turbine combustors, and pollutant dispersion from chimney stacks. Due to these wide range applications, the jet in crossflow (JICF) configuration has been the subject for numerous experimental and theoretical studies. Since the observation of coherent structures in earlier 1970s, various experiments have been devoted to JICF research (see, e.g., Margason [1]) and it was believed, based on those studies, that the dynamic process of flow motions in the JICF is closely relevant to and, at some extent, predominated by the formation and evolution of large vortical structures, which are originated from the jet shear layer, evolved after the jet exit, and decayed further downstream. Fric and Roshko [2] identified four different vortical structures presented in a JICF system for the first time and pointed out that the counterrotating vortex pair (CRVP) is the dominant vortical structure in this kind of complex flow systems.

Despite the fact that the inclined jet and jet with various hole exit shape have been widely used for the blade film cooling by gas turbine and aeroengine industries, there are relatively few studies focusing on the understanding of the underlying flow physics. In case of the inclined jet, earlier experimental work by Lee et al. [3] revealed that significant flow structure changes happened at an inclined jet angle of $35^{\circ}$. Yuen and Martinez-Botas [4] carried out a systematic experimental study at three jet inclination angles of $30^{\circ}, 60^{\circ}$, and $90^{\circ}$ and identified the maximum cooling effectiveness, which was reached at a velocity ratio of 0.33 and jet angle of $30^{\circ}$. Similarly, the jet exit hole shape also plays an important role in determining the adiabatic cooling effectiveness and the cooling boundaries that are often used as a measure on how blade surface could be protected $[5,6]$. Due to a "lifted-off" effect of counterrotating vortex pair (CRVP) in the near field of a hole exit, the "cooled" jet flow tends to be "separated or detached" from the blade surface and this will cause the significant reduction of the cooling effectiveness. 


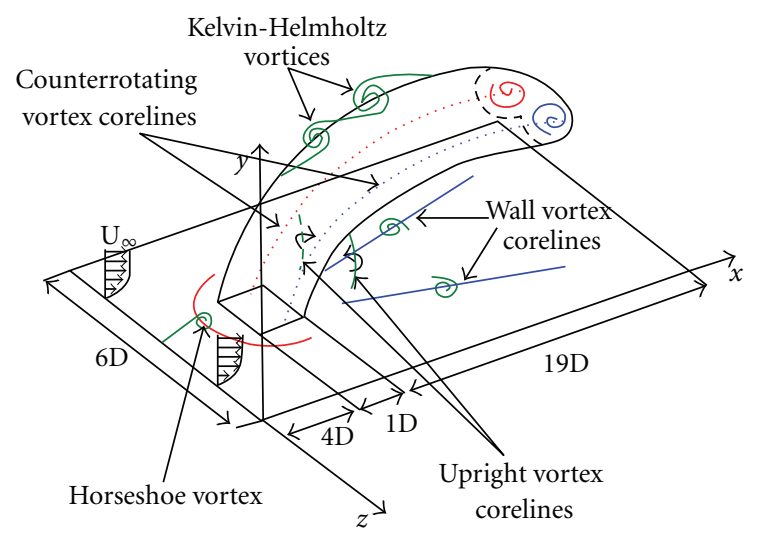

FIGURE 1: A schematic view of large vortex structures in single JICF.

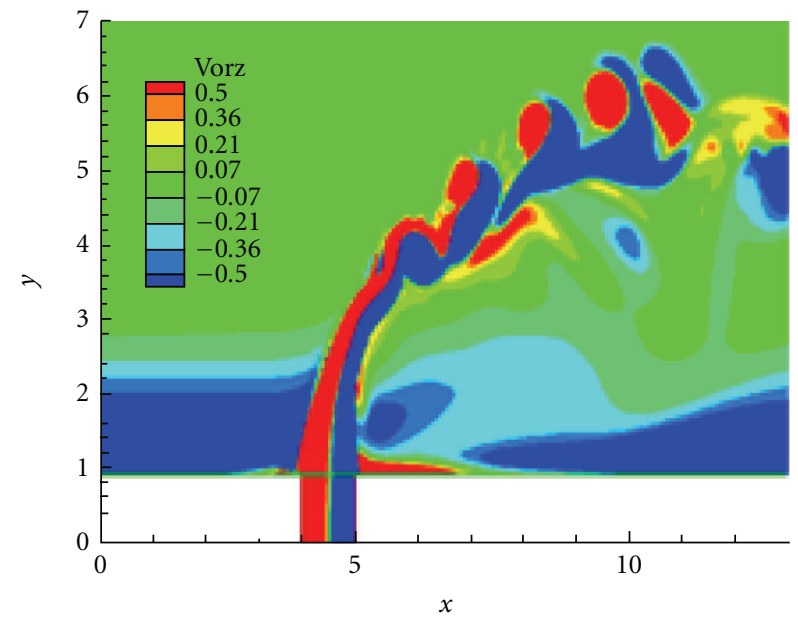

(a)

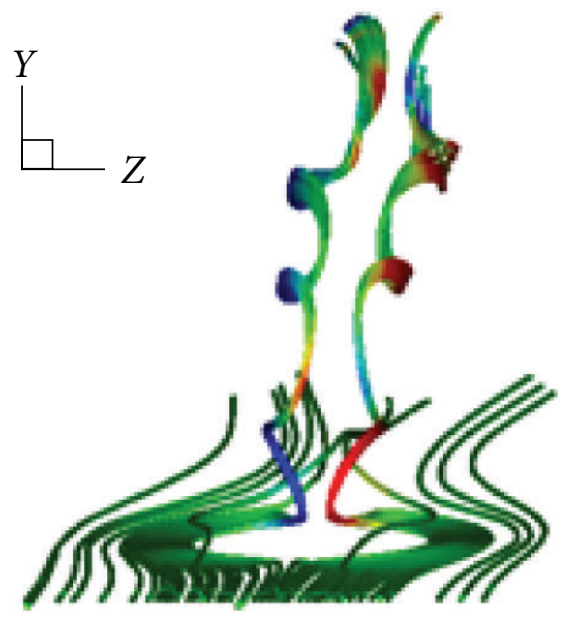

(b)

FIgURe 2: Normal single square jet in cross-flow. (a) Contours of spanwise vortices $\omega_{z}$ on a vertical plane and (b) 3D streamlines showing formation of Kelvin-Helmholtz vortex systems.

However, some flow physics related to the underlying cooling mechanism is still not clear yet.

The present paper focuses on the investigation of the effect of different jet inclination angles and hole geometries on the dynamic evolution process of vortical structures associated with jet and cross-flow interactions using direct numerical simulation (DNS) approach. A parallel in-house DNS solver $[7,8]$ will be used to simulate the vortical flow development around square, round, and elliptic jets issuing normally into a cross-flow.

\section{Numerical Method}

In the present numerical paper, the compressible timedependent three dimensional Navier-Stokes and energy equations are solved. By using reference values at free stream, the nondimensional form of these equations can be written as

$$
\begin{gathered}
\frac{\partial \rho}{\partial t}+\frac{\partial \rho u_{j}}{\partial x_{j}}=0, \\
\frac{\partial \rho u_{i}}{\partial t}+\frac{\partial \rho u_{i} u_{j}}{\partial x_{j}}=-\frac{\partial p}{\partial x_{i}}+\frac{1}{\operatorname{Re}} \frac{\partial \tau_{i j}}{\partial x_{j}}, \\
\frac{\partial E}{\partial t}+\frac{\partial(E+p) u_{j}}{\partial x_{j}}=\frac{\partial u_{i} \tau_{i j}}{\partial x_{j}}+\frac{1}{(\gamma-1) \operatorname{Re} \operatorname{Pr}^{2}} \frac{\partial}{\partial x_{j}}\left(\mu \frac{\partial T}{\partial x_{j}}\right),
\end{gathered}
$$

where $\tau_{i j}$ represents shear stress tensor, Re the Reynolds number, $\gamma$ the specific heats, Pr the Prandtl number, $M$ the 

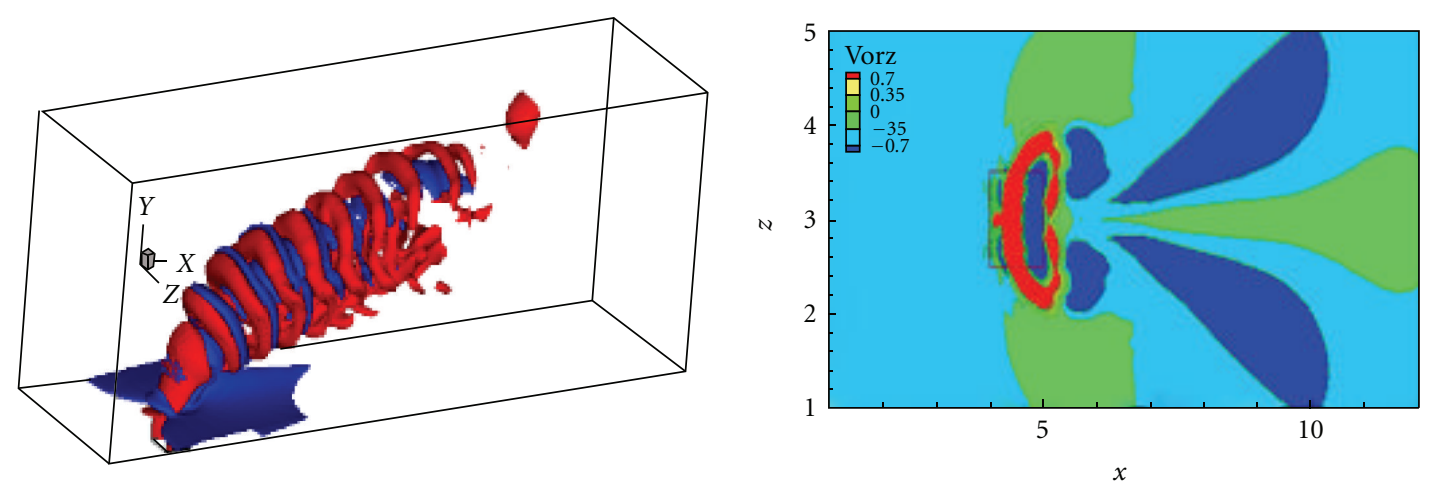

(a) Normal square jet at $90^{\circ}$ angle
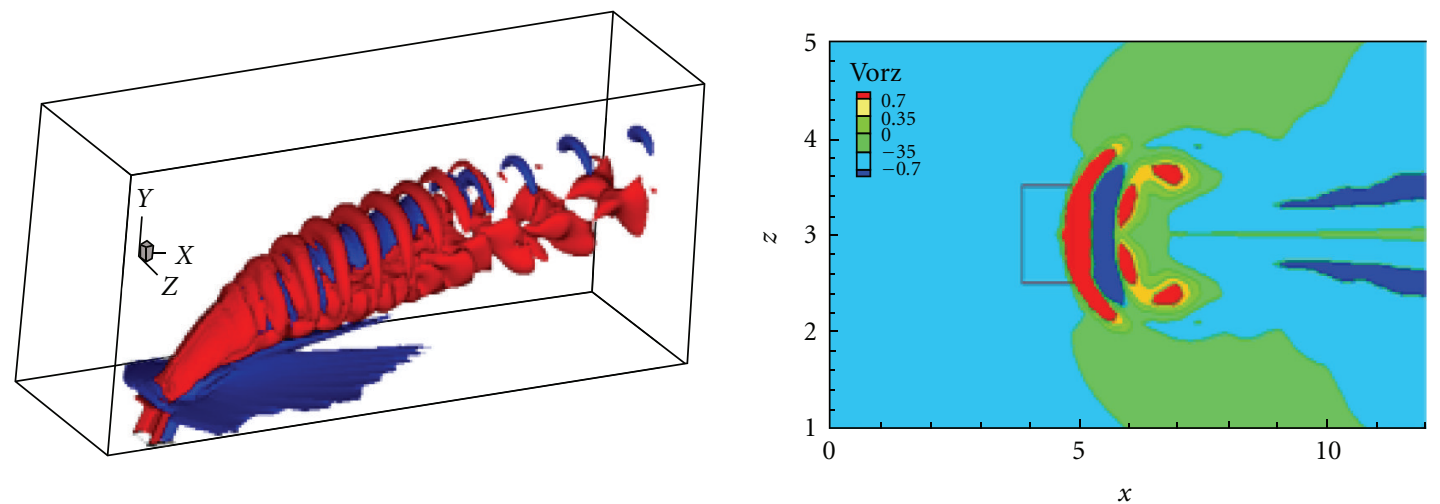

(b) Inclined square jet at $60^{\circ}$ angle
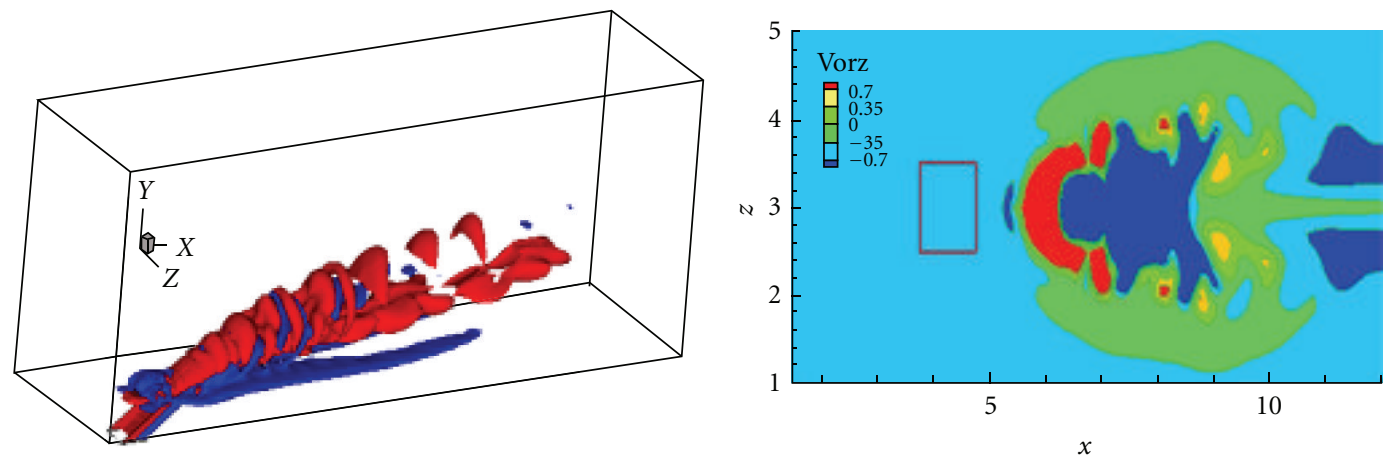

(c) Inclined square jet at $30^{\circ}$ angle

Figure 3: Simulated 3D isosurfaces of the spanwise vorticity $\left(\omega_{z}=0.5 u^{2} / \mathrm{D}^{2}\right.$, red: $\omega_{z}=+0.5$, blue: $\left.\omega_{z}=-0.5\right)$ (left) and 2D contours of the spanwise vorticity at $y=1.2 \mathrm{D}$ (right).

Mach number, and $\mu$ the dynamic viscosity. All other variables follow the standard notations as seen in Yao et al. [9]. The code was developed for compressible flow. In this application, we adopt low Mach number $(\sim 0.2)$ to reduce the compressibility effects.

The code has been parallelized using the MPI library and validated extensively for numerous configurations including laminar boundary layer and channel flows, turbulent boundary layer, turbulent channel flows, transonic flow over a bump geometry with shock/boundary layer, and jet in crossflow. Recent code reengineering work has made it more robust and efficient [10].

\section{Problem Definitions}

The problem considered for simulations consists of a baseline configuration of a single square jet issuing perpendicularly into a main cross-flow field with two further scenarios, that is, (1) square jets issuing at an inclination angle of $60^{\circ}$ and $30^{\circ}$ against the horizontal walls and (2) round and elliptic jet hole shape issuing at a normal $90^{\circ}$ angle against incoming cross-flow, respectively.

The computational box (baseline) has a cross-flow domain of $24 \mathrm{D} \times 8 \mathrm{D} \times 6 \mathrm{D}$ in the streamwise, the wall normal and the lateral directions, respectively, and a jet domain of 

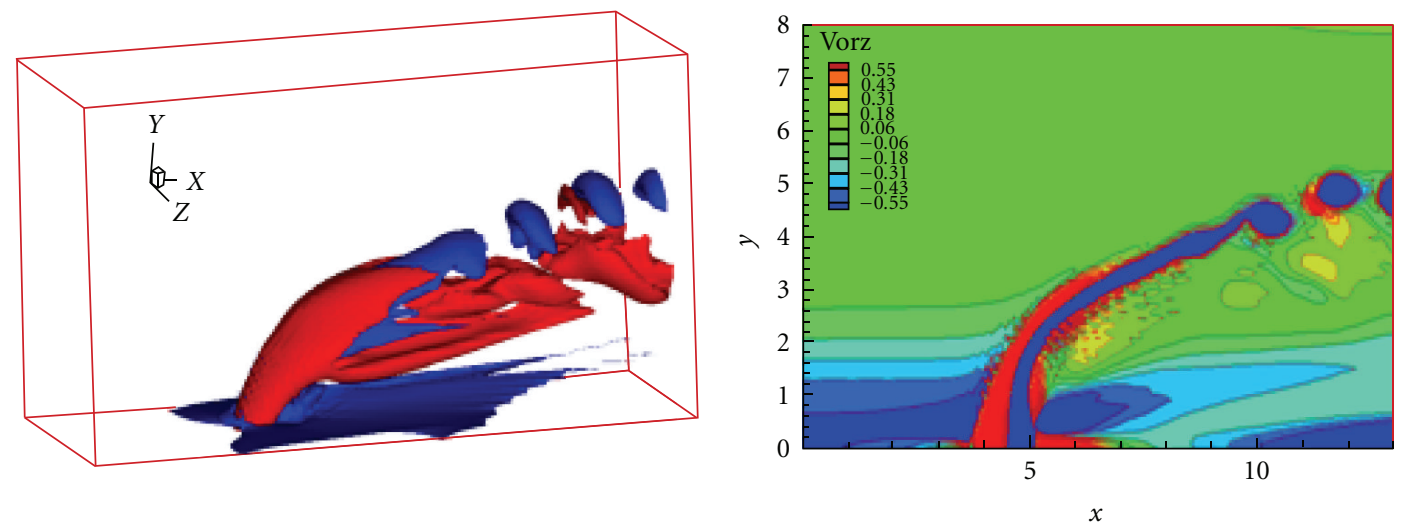

(a) Round jet
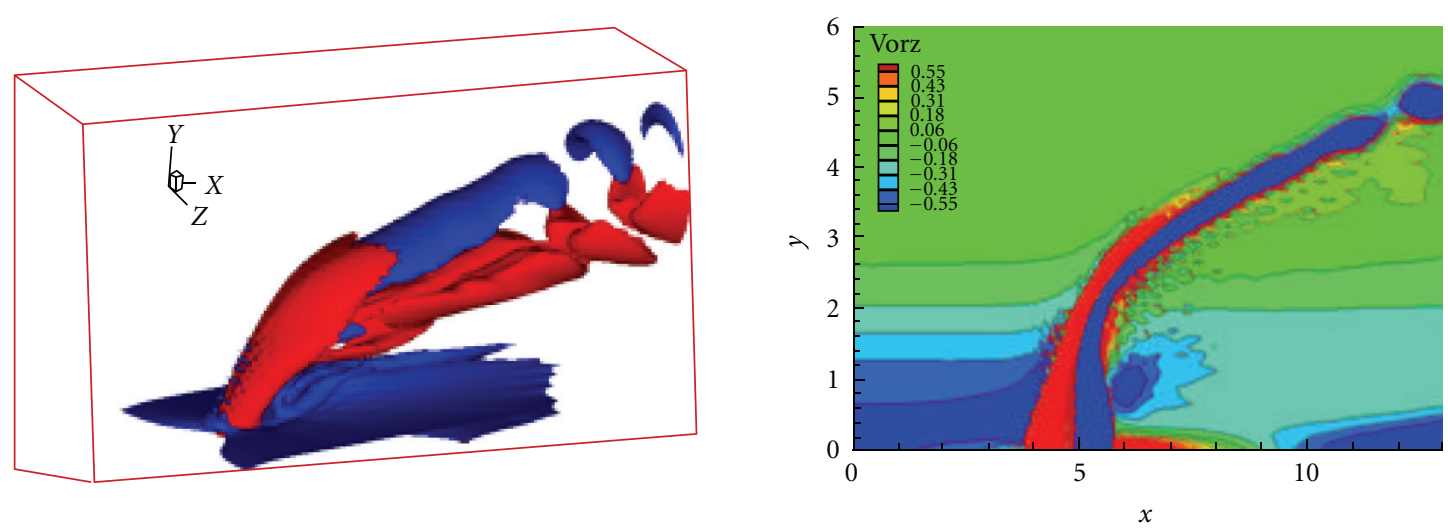

(b) Elliptic jet

FIGURE 4: Simulated isosurfaces of the spanwise vorticity $\left(\omega_{z}=0.5, u^{2} / \mathrm{D}^{2}\right.$, red positive and blue negative) (left) and vorticity contours at midplane (right).

$1 \mathrm{D} \times 1 \mathrm{D} \times 1 \mathrm{D}$, where $\mathrm{D}$ is the jet width. In case of circulartype jets, the jet hole exit area is kept the same as the square jet case and then the radius of round jet and the major and minor axis length of elliptic jet (assuming a ratio of 2) can be derived accordingly. In case of square jet, it is located in $4 \mathrm{D}$ and $5 \mathrm{D}$ from the cross-flow inlet plane in the streamwise direction and 2.5D and 3.5D in the spanwise direction, respectively (see Figure 1). Following a previous numerical study of Sau et al. [11], a computational grid of 241 points in the streamwise (with 11 points in the jet domain), 81 points in the wall normal, and 61 points in the spanwise direction (with 11 points in the jet domain) is adopted. Both domain influence and grid refinement studies have been carried out [12]. For example, simulations were performed on domain sizes of $24 \mathrm{D} \times 9 \mathrm{D} \times 8 \mathrm{D}$ and $24 \mathrm{D} \times$ $6 \mathrm{D} \times 12 \mathrm{D}$ (using similar grid resolutions and distributions) and computational meshes of $192 \times 65 \times 49$ and $361 \times 121 \times$ 91 (both applied to the baseline domain), respectively. The examination of streamwise and vertical velocity profiles at representative locations of the cross-flow domain has shown that very small differences were generated by the domain size increases and the grid refinements. It was therefore decided to retain both the baseline domain and the grids as described above for the flow analysis simulations.
The cross-flow velocity profile is initialized using a similarity solution of laminar boundary layer at Reynolds number of 225. A standard circular pipe flow Poiseuille-type velocity profile, that is, $v(r)=v_{\max }\left(1-r^{2} / R^{2}\right)$, is used at the inlet of a circular jet orifice and for 2D non-circular duct and elliptic pipe geometries, an interpolation procedure is applied. The characteristics boundary conditions are used at both outlet plane and upper surface and periodic conditions for the side planes. Simulations are performed for a jet to cross-flow velocity ratio $R=2.5$ and the Reynolds number $\operatorname{Re}=225$, based on the free-stream quantities of the crossflow and the jet width (D) and the Mach number $M=0.2$, based on the free-stream quantities.

\section{Results and Discussions}

Similar to Sau et al. [11], results presented here were taken at an instantaneous dimensionless time unit of $t=18$, as the main objective of the study is to visualize the vortex flow structures. Discussions will focus on understanding the underlying flow physics in JICF at different scenarios.

Figure 2(a) depicts contours of spanwise vorticity $\left(\omega_{z}\right)$ at a midspan plane $(z=0)$ and it reveals the presence of the shear layer vortex that emerges at the upstream side 

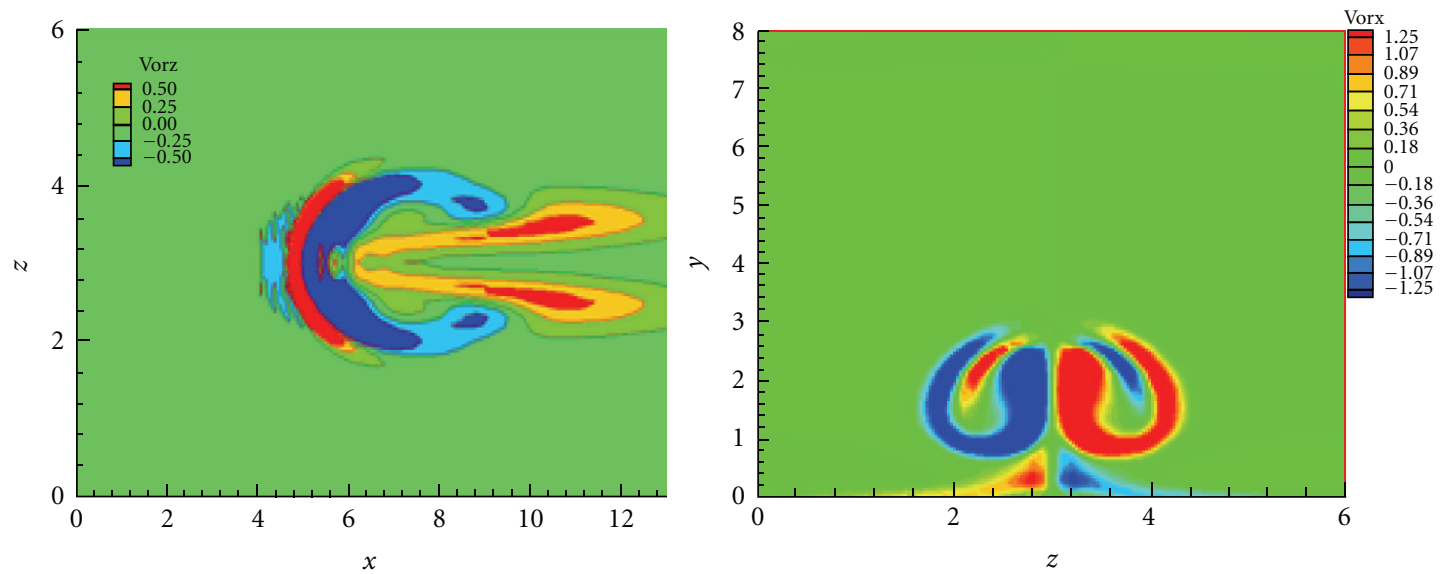

(a) Square jet
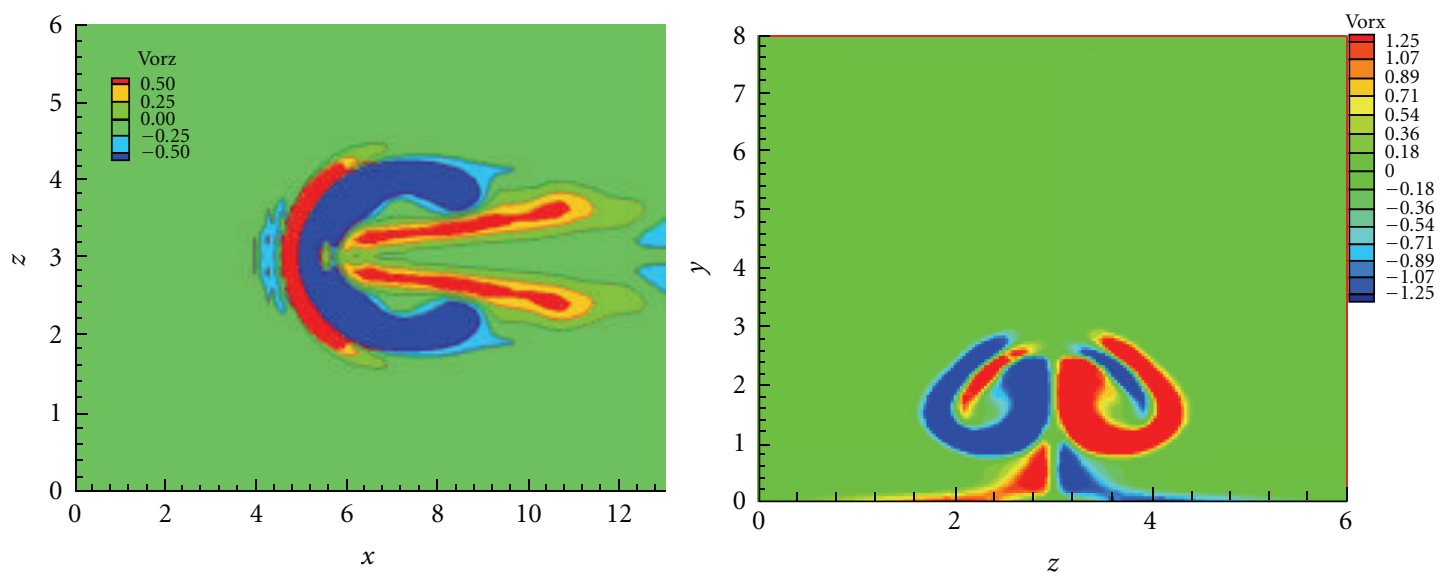

(b) Round jet
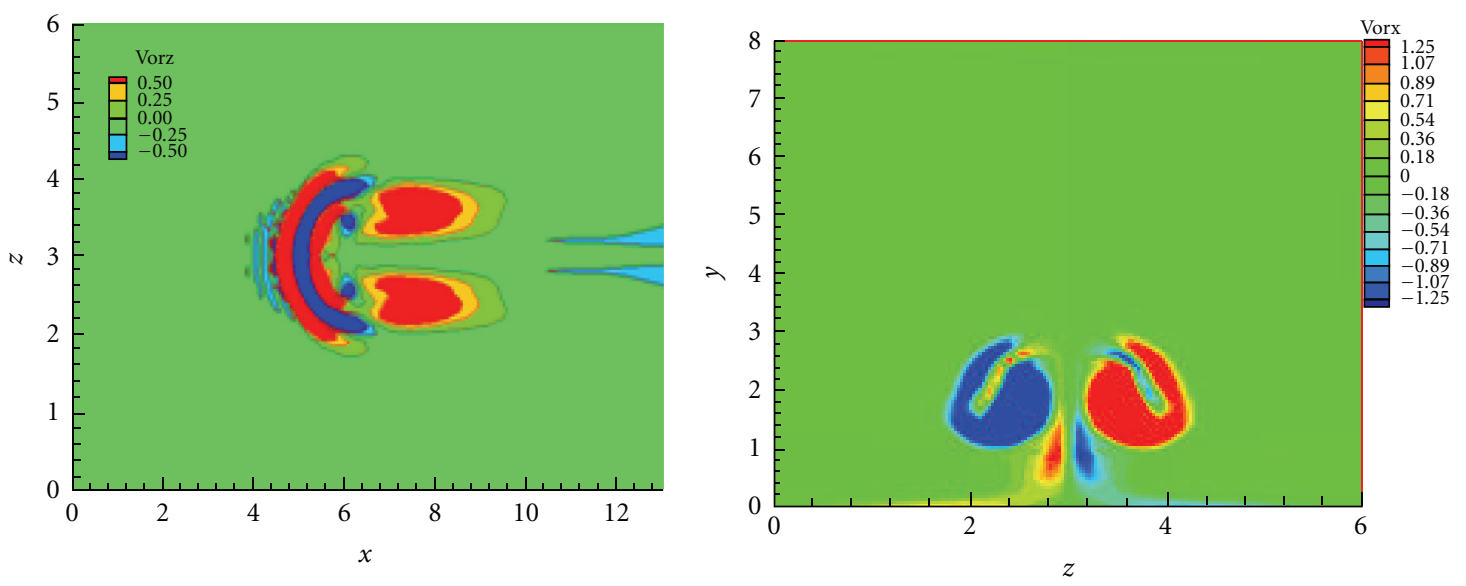

(c) Elliptic jet

Figure 5: Simulated $\omega_{z}$ contours at $y=1.8 \mathrm{D}$ (left) and $\omega_{x}$ contours at $x=6.5 \mathrm{D}$ (right).

of the initial portion of the jet. The roll-up process of the shear layer upstream of the jet has been attributed to the Kelvin-Helmholtz (K-H) instabilities [2, 11, 13]. Figure 2(b) reveals the evolution of the three-dimensional streamlines participating in the formation of a K-H vortex system in the near-wake region that emerges at the upstream (wind side) of the initial portion of the jet flow. This type of rollup process of the shear layer upstream of the jet has been attributed to the so-called Kelvin-Helmholtz instabilities as discussed in previous studies (e.g., Fric and Roshko [2], Smith and Mungal [14]). Due to the merging process whilst the vortex roller moving downstream, the scale of $\mathrm{K}-\mathrm{H}$ 
vortices increases with the distance, similar to that in a free jet flow. Overall present observations are in good agreement with previous experimental and numerical predictions [12].

The influence of the streamline injection angle variations on the flow structures in jet cross-flow interaction has been assessed. Figure 3 presents the isosurfaces of spanwise vorticity $\left(\omega_{z}\right)$ for $90^{\circ}, 60^{\circ}$, and $30^{\circ}$ injection angles (left). The CRVP is clearly present in all cases of injection angle with the shear layer (Kelvin-Helmholtz) vortices emerge at the upstream side of the initial portion of the jets. However, for the $30^{\circ}$ injection case, as the jet centerline is much closer to the wall, the evidence of Kelvin-Helmholtz rollers is less clear. As injection angle increases, the jet penetration is higher and the spreading area is wider. The vorticity contours of $\omega_{z}$ are shown at location $y=1.2 \mathrm{D}$ from the wall in Figure 3 (right). Compared with the normal injection case at the same blowing ratio, the starting position of the CRVP is shifted downstream and its strength is reduced at both $60^{\circ}$ and $30^{\circ}$ injections. For the $30^{\circ}$ injection, since the jet is much closer to the wall, the interaction between the jet and the boundary layer is more complex in the wake region. The evidence of wake vortices is not as clear as in the instantaneous plots in Figure 3(c) (right).

The study continues with the effect of jet exit hole shape variation with complicated nature of the JICF illustrated. Figure 4 depicts 3D isosurfaces of the spanwise vorticity and its $2 \mathrm{D}$ contours on the symmetric plane $(x, y, z=L z / 2)$, that exhibit clear rollup of vortical structures, in addition to the tilting of the evolving vortex rings. The delayed rollup of vortical structures observed in the round and elliptic jets, in comparison to that of square jet, is probably due to the removal of the sharp corners, otherwise it will facilitate the earlier formation of vortex structures. Comparing to elliptic jet, the round jet has shown that the vortex rings form earlier downstream. This again could be attributed to the effect of jet orifice shape change. Note that the nature and the dynamics of these coherent structures play a significant role in the mixing and heat transfer process between the jet flow and the cross-flow. Figure 5 (left) presents the spanwise $\left(\omega_{z}\right)$ vorticity contours in the $(x, y=1.8 \mathrm{D}, z)$ plane for the three simulation cases. A different flow structure can be noted between the elliptic jet and that of circular and square jets. This is mainly due to the difference in vortex pairing process (see, e.g., Maidi and Yao [15]) which occurs further downstream from the jet orifice. It is important to recall that the presence of vortex pairing and the formation of kidneyand antikidney-shaped vortices depend on the aspect ratio of the elliptic jet [16]. Figure 5 (right) shows the lower-deck vortex pair (the large contrarotating vortex pair) appearing near the surface of the plate in all cases. The origin of these vortices is the lateral spillage and rollup of the jet sidewall boundary layer. On the top of the lower-deck vortex pair, an opposing upper-deck vortex pair (the small vortex pair) can be identified. The upper-deck vortex pair is the hole leading-edge boundary layer. Both lower- and upper-vortex pairs for a round hole were previously reported by other authors (e.g., Margason [1], Tyagi and Acharya [17]). These vortices have a sense of rotation that promotes both the jet "lifted-off" and "entrainment" of the cross-flow towards the wall, respectively. Thus they can seriously degrade the effectiveness of the protecting film layer near the surface. Based on Figure 5 (right), the jet "lifted-off" is more important in the elliptic jet hole case in which the upperdeck vortex pair, promoting the entrainment of the crossflow toward the wall surface, is relatively weak compared to those identified in the other two hole geometry cases. The results reported here are in good qualitative agreement with the experimental studies of Haven and Kurosaka [16], New et al. [18], and DNS of Sau et al. [11].

\section{Conclusions}

The interactions between jet flow and cross-flow are examined by using a direct numerical simulation approach. Simulation considers two scenarios, including single square jet at three issuing angles of $30^{\circ}, 60^{\circ}$, and $90^{\circ}$ and single circular jet with round and elliptic hole exit geometries.

For single normal jet, the resulting vortex structures were in good agreement with both experiments and other numerical simulations in the literature. The computations successfully reproduced the important vortex patterns presented in this type of flow, including the counterrotating vortex pair (CRVP), horseshoe vortex, shear layer vortex, and wake vortex.

For single inclined jet at $30^{\circ}$ and $60^{\circ}$ angles, simulation results reveal the significant flow structure changes, with a local recirculation flow observed upstream of the jet orifice for a small injection angle of $30^{\circ}$. The vorticity contours also exhibit complicated flow patterns at two inclined jet cases, indicating strong $3 \mathrm{D}$ effects. The jet inclination decreases the lateral deflection of cross-flow.

For circular normal jet, it shows that the hole geometry has considerable influences on the near field characteristics of vortex structure. The proximity of these counterrotating vortices relative to each other is found to affect both the liftoff of the jet and the entrainment of cross-flow fluid toward the wall surface. The obtained results show that the jet liftoff as well as the fluid entrainment into the near wall region mechanisms depends on the hole geometry, in agreement with previous experimental observations in the literature. The maximum jet liftoff is found in the case of elliptic hole geometry.

\section{Acknowledgment}

The support of the UK Engineering and Physical Science Research Council (EPSRC) through the research Grant (EP/ C014979/01) is gratefully acknowledged.

\section{References}

[1] R. J. Margason, "Fifty years of jet in cross flow research," AGARD-CP-534, 1. 1-1. 41, 1993.

[2] T. F. Fric and A. Roshko, "Vortical structure in the wake of a transverse jet," Journal of Fluid Mechanics, vol. 279, pp. 1-47, 1994.

[3] S. W. Lee, J. S. Lee, and S. T. Ro, "Experimental study on the flow characteristics of streamwise inclined jets in crossflow on 
flat plate," Journal of Turbomachinery, vol. 116, no. 1, pp. 97105, 1994.

[4] C. H. N. Yuen and R. F. Martinez-Botas, "Film cooling characteristics of a single round hole at various streamwise angles in a crossflow: part I effectiveness," International Journal of Heat and Mass Transfer, vol. 46, no. 2, pp. 221-235, 2003.

[5] X. Z. Zhang and I. Hassan, "Numerical investigation of heat transfer on film cooling with shaped holes," International Journal of Numerical Methods for Heat and Fluid Flow, vol. 16, no. 8, pp. 848-869, 2006.

[6] J. Yao and Y. F. Yao, "Computational study of hole shape effect on film cooling performance," Proceedings of the Institution of Mechanical Engineers A, vol. 225, no. 4, pp. 505-519, 2011.

[7] Y. F. Yao, A. A. Lawal, N. D. Sandham, I. C. Wolton, M. Ashworth, and D. R. Emerson, "Massively parallel simulation of shock/boundary-layer interactions," in Proceedings of International Conference Applied Computational Fluid Dynamics, pp. 728-735, Beijing, China, 2000.

[8] N. D. Sandham, Y. F. Yao, and A. A. Lawal, "Large-eddy simulation of transonic turbulent flow over a bump," International Journal of Heat and Fluid Flow, vol. 24, no. 4, pp. 584-595, 2003.

[9] Y. F. Yao, L. Krishnan, N. D. Sandham, and G. T. Roberts, “The effect of Mach number on unstable disturbances in shock/ boundary-layer interactions," Physics of Fluids, vol. 19, no. 5, Article ID 054104, 2007.

[10] Y. F. Yao, Z. Shang, J. Castagna et al., "Re-engineering a DNS code for high-performance computation of turbulent flows," in Proceedings of the 47th Aerospace Sciences Meeting Including the New Horizons Forum and Aerospace Exposition ( AIAA '09), Orlando, Fla, USA, January 2009.

[11] A. Sau, T. W. H. Sheu, R. R. Hwang, and W. C. Yang, "Threedimensional simulation of square jets in cross-flow," Physical Review E, vol. 70, no. 1, Article ID 019902, 1 pages, 2004.

[12] Y. F. Yao and M. Maidi, "Direct numerical simulation of single and multiple square jets in cross-flow," Journal of Fluids Engineering, Transactions of the ASME, vol. 133, no. 3, Article ID 031201, 2011.

[13] R. M. Kelso, T. T. Lim, and A. E. Perry, "An experimental study of round jets in cross-flow," Journal of Fluid Mechanics, vol. 306, pp. 111-144, 1996.

[14] S. H. Smith and M. G. Mungal, "Mixing, structure and scaling of the jet in crossflow," Journal of Fluid Mechanics, vol. 357, pp. 83-122, 1998.

[15] M. Maidi and Y. F. Yao, "Numerical visualization of vortex flow behavior in square jets in cross-flow," Journal of Visualization, vol. 11, no. 4, pp. 319-327, 2008.

[16] B. A. Haven and M. Kurosaka, "Kidney and anti-kidney vortices in crossflow jets," Journal of Fluid Mechanics, vol. 352, pp. 27-64, 1997.

[17] M. Tyagi and S. Acharya, "Large eddy simulation of film cooling flow from an inclined cylindrical jet," Journal of Turbomachinery, vol. 125, no. 4, pp. 734-742, 2003.

[18] T. H. New, T. T. Lim, and S. C. Luo, "A flow field study of an elliptic jet in cross flow using DPIV technique," Experiments in Fluids, vol. 36, no. 4, pp. 604-618, 2004. 

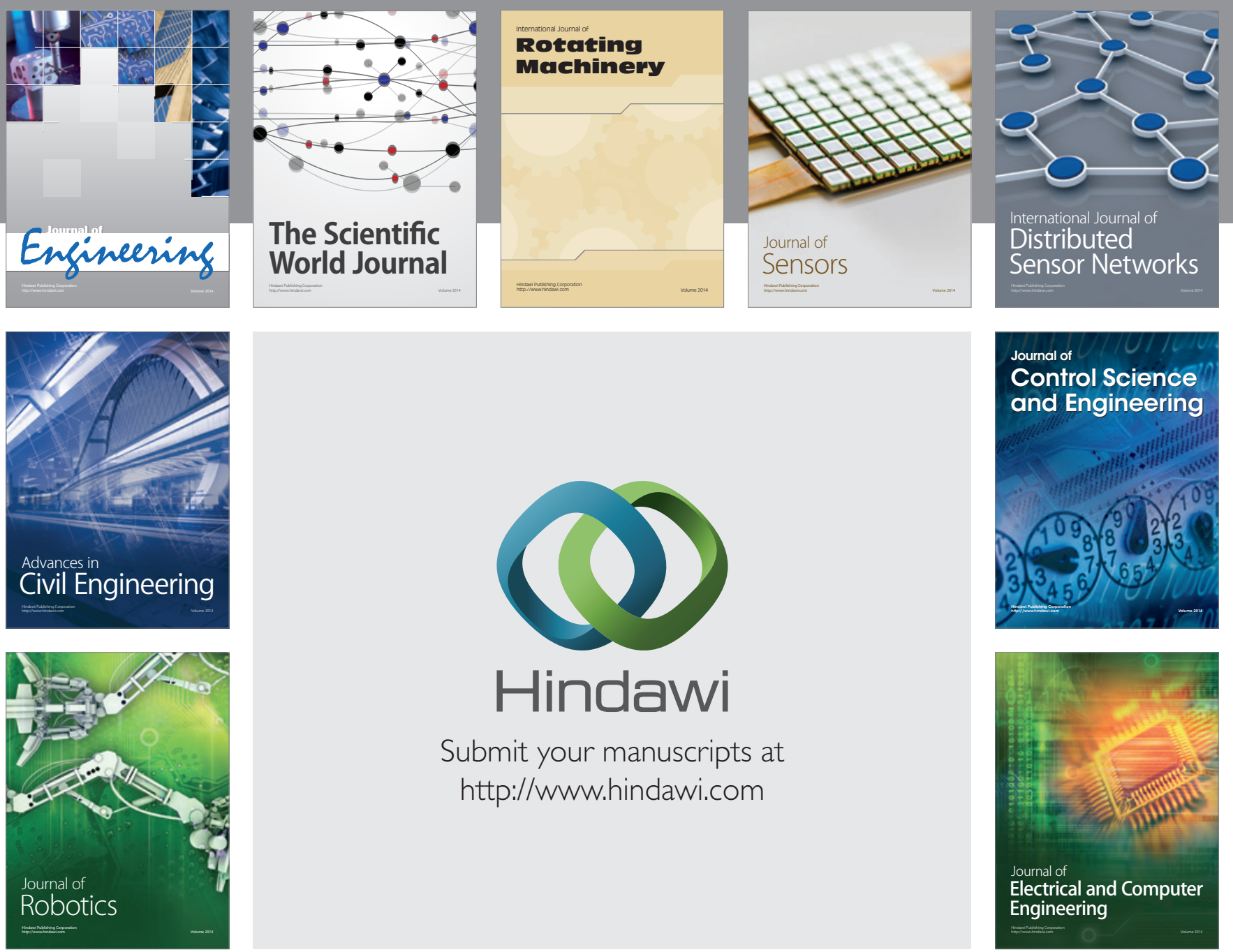

Submit your manuscripts at

http://www.hindawi.com
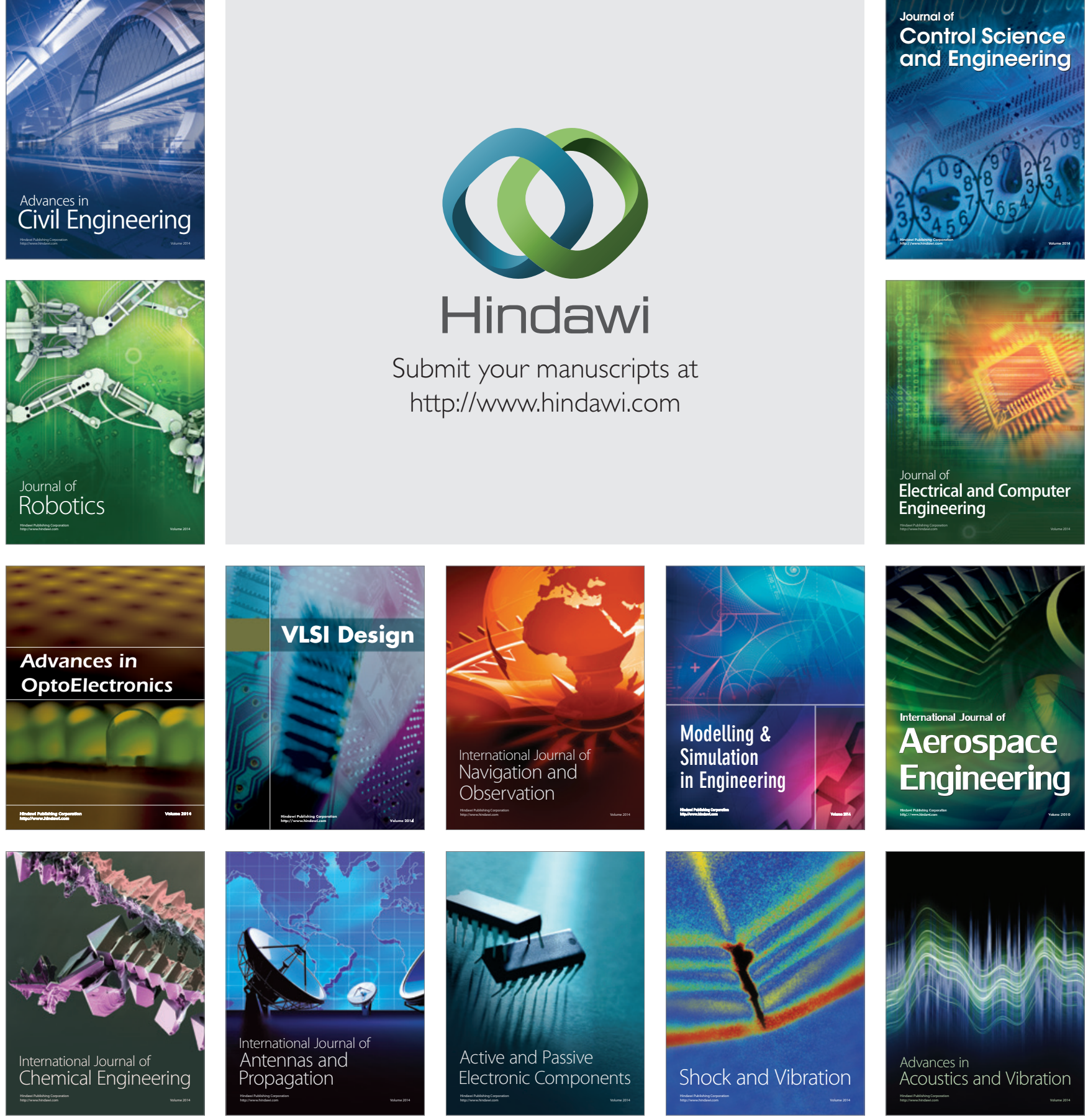